\title{
Concepto y proceso constructivo de la cortina cerámica de la Biblioteca de Vila-real
}

\section{Concept and construction process of the ceramic curtain of Vila-real Library}

\author{
$\underline{\text { A. Peñín Llobell }}^{(*)}$, B. Ferrater ${ }^{(*)}$

\section{RESUMEN} \\ La construcción de la Biblioteca de Vila-real, edificio seleccionado en 2012 en la VIII Bienal Iberoamericana de Arquitectu- \\ ra y Urbanismo, pone de manifiesto la importancia de la colaboración con la industria para el desarrollo y puesta en obra de \\ su membrana exterior. El análisis del proceso constructivo de la cortina cerámica que la define, realizada mediante piezas \\ cilíndricas de cerámica vidriada blanca de altura $7,5 \mathrm{~m}$ y diámetro $5 \mathrm{~cm}$, así lo desvela. El sistema construye un espacio \\ intersticial imprescindible para el funcionamiento e integración medioambiental del edificio, y, al tiempo, lo vincula tanto \\ al tejido local industrial, del que aspira a erigirse como uno de sus exponentes, como a la cultura mediterránea de filtros y \\ cortinas de eslabones. El procedimiento seguido se adscribe a la visión pragmática que, superado el movimiento moderno, \\ implantaron personajes como Jean Prouvé y que, defendemos, hoy debiera encontrar cauces naturales y legales para su \\ desarrollo en aras del progreso del sector de la construcción.
}

Palabras clave: arquitectura; industria; cortina cerámica; Vila-real; biblioteca; Prouvé; OAB.

\section{ABSTRACT}

The construction of the Library of Vila-real, selected in 2012 in the VIII Latin American Biennial of Architecture and Urbanism, highlights the importance of collaboration with industry for the development and application of its outer membrane. The analysis of the construction process of the ceramic cylindrical curtain that defines it, performed by white glazed ceramic, $5 \mathrm{~cm}$ diameter and $7.5 \mathrm{~m}$ height, reveals this fact. The system builds an interstitial space, essential for its use and environmental integration. At the same time it links the building to both local industrial fabric, which aims to establish itself as one of its exponents, and to the Mediterranean culture of filters. The procedure followed is ascribed to the postartesanal and pragmatic perspective that beyond the modern heroes, introduced characters like Jean Prouvé and that today, we state, should find natural and legal channels for its development, on behalf of the progress of the construction sector.

Keywords: architecture; industry; ceramic curtain; Vila-real; library; Prouvé; OAB.

(*) Arquitecto, Profesor Agregado SH, UPC.

(**) Arquitecto, Profesor UIC.

Persona de contacto/Corresponding author: alberto@penin.es (A. Peñín Llobell).

Cómo citar este artículo/Citation: Peñín Llobell, A., Ferrater, B. (2016). Concepto y proceso constructivo de la cortina cerámica de la Biblioteca de Vila-real. Informes de la Construcción, 68(544): e166, doi: http://dx.doi.org/10.3989/ic.15.157.m15

Copyright: (C) 2016 CSIC. Licencia / License: Salvo indicación contraria, todos los contenidos de la edición electrónica de Informes de la Construcción se distribuyen bajo una licencia de uso y distribución Creative Commons Attribution License (CC BY) Spain 3.o. 


\section{INTRODUCCIÓN}

No creo en la claridad desde el inicio, en un proyecto cerrado y prefijado. Es preferible otear lo desconocido.

Jean Prouvé (1).

El autor de esta cita, el creador y constructor francés Jean Prouvé (1901-1984), representa la superación de la distancia entre arquitectura e industria que el movimiento moderno, pese a su sueño de taylorización de la construcción, no pudo resolver y cuya vigencia quisiéramos analizar en este texto a través del análisis de un proyecto contemporáneo. Prouvé, desde una óptica postartesanal ${ }^{1}$, establecía una nueva operativa del proyecto donde la secuencia de definición arquitectónica se basa en un proceso colectivo de colaboración con la industria. Laimplantada afecta no slo a la producción, sino también a la organización del trabajo, estrategia necesaria para enfrentarse a los retos abiertos del proyecto. Su desarrollo obedece a un procedimiento iterativo desarrollado de la mano del tejido industrial, que pasa directamente del boceto al prototipo, corregido y mejorado, tras el cual se traza el plan de ejecución. El proceso, cuyo escenario fundamental es la obra, se nutre del trabajo de equipo y del estímulo de la iniciativa individual por encima del trabajo mecánico y sectorizado. El resultado de esta colaboración entre arquitectura e industria es, contrariamente a lo que pudiera parecer, 'antiindustrial'. Así lo muestra no sólo su obra y su pensamiento, sino también edificios como el centro Pompidou ${ }^{2}$, surgidos de un proceso desarrollado en la obra y que extienden el tiempo de la concepción a la propia materialización del proyecto.

Esta suerte de empirismo artesanal, que integra la industria en el proceso de definición de la arquitectura, es el que guía la concepción y el proceso de construcción del proyecto de la Biblioteca municipal de Vila-real, y en particular el de su cortina cerámica. Surgido de un concurso ganado en 2009, y seleccionado en la VIII Bienal Iberoamericana de Arquitectura y Urbanismo, el proyecto concebido por OAB y Peñín Arquitectos con la colaboración en su ejecución de GyF Arquitectura $^{3}$, se sitúa en plena cuenca azulejera castellonense. El proceso constructivo no slo se desarrollará de la mano de una de las empresas punteras del sector, sino que además expresa esta colaboración a través de una imagen contemporánea y de alta tecnología que recoge la herencia cultural e industrial de la zona, para aspirar a convertirse en uno de sus exponentes.

\section{EL PROYECTO}

La Biblioteca municipal de la ciudad de Vila-real, en la plana de Castellón, se implanta sobre la estructura de un aparcamiento existente con unas limitadas condiciones técnicas y geométricas. El solar de forma rectangular de $40 \times 17$ $\mathrm{m}$, orientado en su lado largo hacia el Noroeste, consta de $4.685 \mathrm{~m}^{2}$ (Figura 1).

El volumen propuesto, un sencillo prisma de planta baja y piso de $83 \times 17 \mathrm{~m}$ por 9 de altura, integra las escaleras e instalaciones del parking preexistente. Sus requerimientos técnicos quedan subordinados a las posibilidades que ofrece esta estructura previa, sobre la cual la nueva construcción se ha de apoyar. La disposición del edificio libera una plaza vinculada al colegio adyacente, cuya urbanización se proyecta extendiendo deliberadamente los límites del proyecto. Esta colocación asimétrica permite ofrecer una fachada alineada a la avenida principal Pío XII, subrayando así el carácter urbano de la pieza en la ciudad de Vila-real, una ciudad de fundación y de traza ordenada.

La estructura interior del prisma organiza una secuencia funcional de espacios de distinto tamaño y clara geometría, que
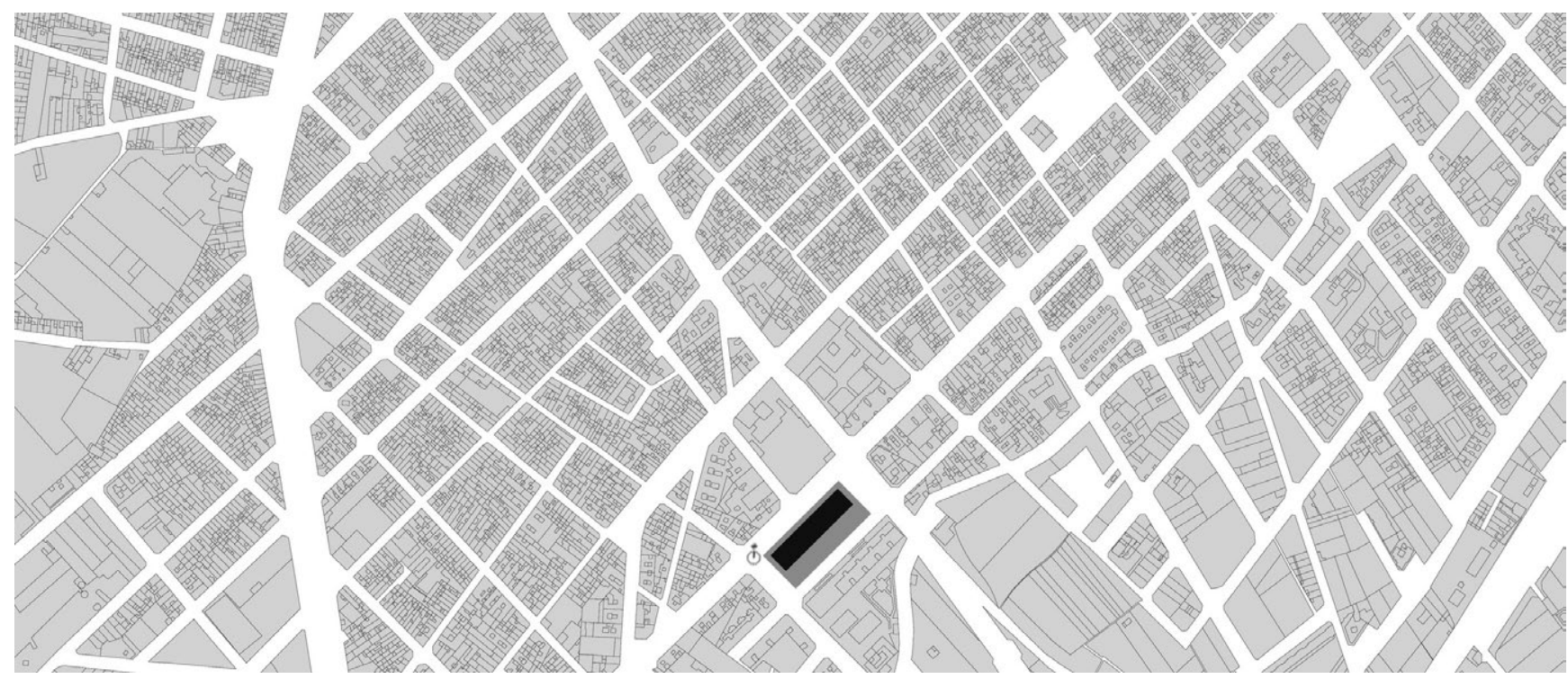

Figura 1. Plano de emplazamiento. Elaboración propia.

\footnotetext{
${ }^{1}$ Implica la adquisición por parte de la industria de una dimensión ética más allá del objeto, tal y como se define en Jean Prouvé constructeur (2), pág. 14.

${ }^{2}$ Prouvé fue presidente del jurado y fue el valedor, así como profesor en l'Ecole des Ponts et Chaussées, de uno sus arquitectos, Renzo Piano.

${ }^{3}$ El proyecto está firmado por Carlos Ferrater, Alberto Peñín y José Luis Gimeno como arquitectos. Aparejadores E. Fabregat y E. Mesquida e ingeniero de instalaciones J. Dolz.
} 


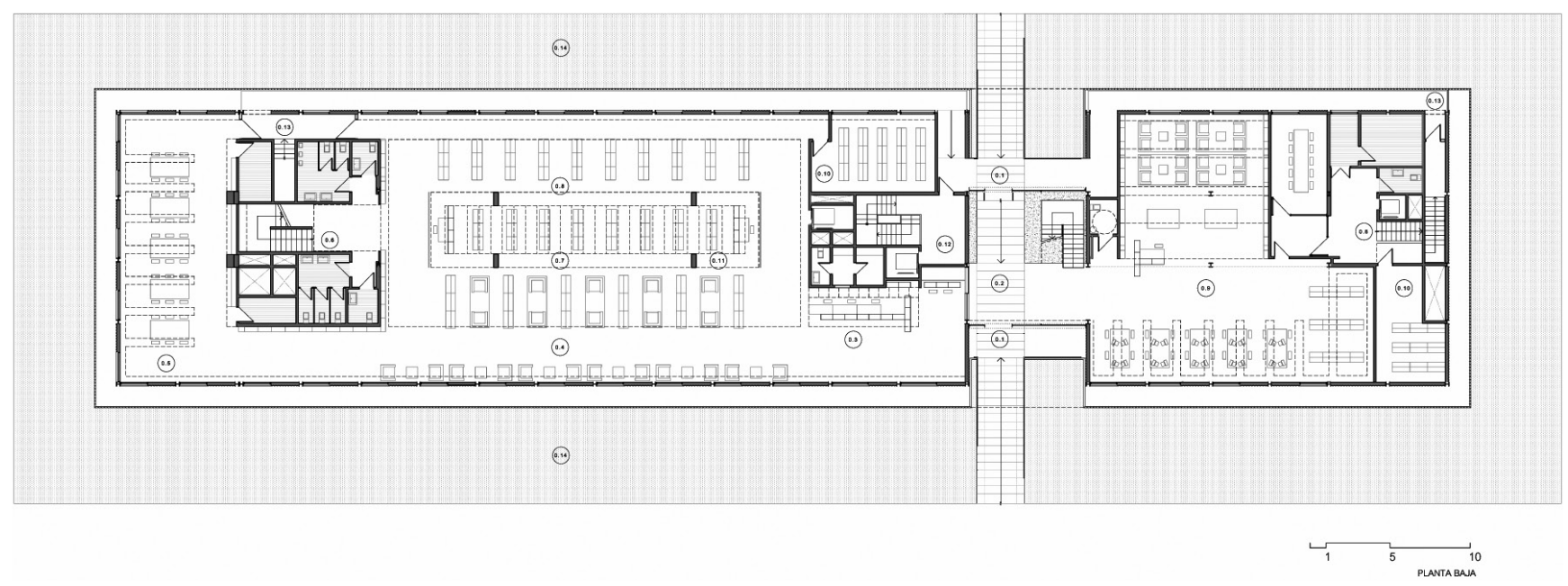

Figura 2. Planta baja. Elaboración propia.

admiten distintas configuraciones en función de los criterios que adopte la dirección de la Biblioteca, sin necesidad de recurrir a compartimentación alguna. Podrá albergar hasta 75.00o libros y 275 puestos de lectura. Se apuesta por la flexibilidad y la creación de espacios de reserva para futuros desarrollos. Dentro de esta diversidad surgen espacios adecuados para la lectura, la consulta o el estudio. Se ha realizado un exhaustivo trabajo con las instalaciones y los techos, buscando la integraciuier tipo de elemento visto y atendiendo a los obligados requerimientos de absorción acústica.

El acceso al inmueble se sitúa aproximadamente a un tercio de la fachada, permitiendo acortar su dimensión y facilitar un acceso pasante. El atrio central de la Biblioteca se transforma en un espacio público más, uniendo la avenida Pío XII con la plaza-jardín frente al colegio (Figura 2).

La profundidad del edificio se enfatiza a través del recorrido por el puente de acceso y el trabajo de una doble fachada. El prisma descansa sobre un talud vegetal que dispone el edificio sobre un podio. La secuencia del exterior al interior se realiza a través de una serie de filtros en los que la cortina cerámica adquiere un gran protagonismo.

\section{EL FILTRO 4}

La sencillez del planteamiento, su clara volumetría y disposición en el solar otorgan un papel preponderante a las cualidades materiales del edificio. La contundencia formal induce inversamente un tratamiento sutil y desmaterializado del prisma que invita a traspasarlo y a habitarlo en su interior. De manera opuesta la percepción interna del volumen perseguirá una relación con el exterior menos inmediata que la que pudiera reconocerse inicialmente, facilitando además la necesaria intimidad y reclusión que un espacio de lectura y estudio requiere.

Desde estas orientaciones surge la idea de construir un espacio intersticial, con el objetivo añadido de conducir las salidas de emergencia del parking existente a su través. La elabora- ción de la membrana que separa con el exterior adquiere relevancia, y merece la atención prestada tanto en el concurso, como en su ulterior desarrollo constructivo.

El proyecto plantea en este punto una doble contextualización. En primer lugar, una contextualización cultural, que hace referencia a los espacios intermedios de nuestra tradición mediterránea y en particular a algunos de sus límites, elaborados mediante filtros separadores de eslabones de madera que protegen del sol y de los insectos a los umbrales de tantos pueblos próximos al litoral. Se crean espacios de sombra, microclimas, produciendo una sofisticación en las fachadas que el movimiento moderno, en su primera versión funcionalista, no supo integrar.

La reinterpretación contemporánea que se propone de estas ancestrales cortinas implica una segunda adaptación. Una suerte de contextualización constructiva que recurre al tejido industrial de proximidad para su desarrollo, en este caso en la zona de Vila-real (Figura 3), como si de la propia tierra en la que se asienta surgiera el material de la arquitectura.

El desarrollo puntero de su sector cerámico condujo a la integración de este material a través de una fructífera colaboración con una de las empresas de referencia en el sector (Butech), auspiciada por la constructora ${ }^{5}$. Junto a su departamento técnico se desarrolló la cortina y sus eslabones de

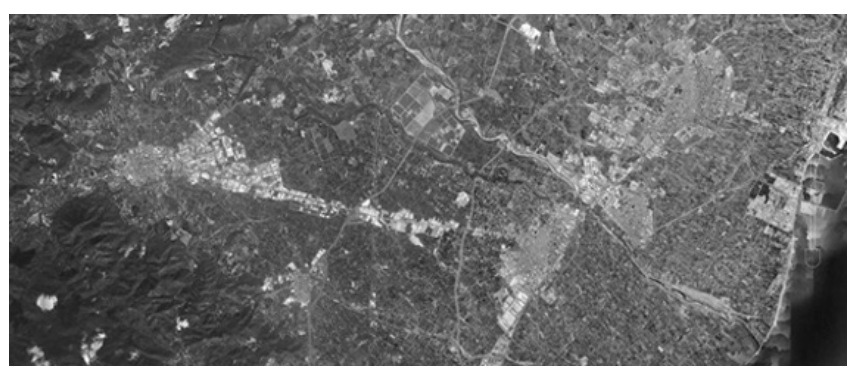

Figura 3. El proyecto y su territorio. Cuenca azulejera de la plana de Castellón. El eje Vila-real/Onda. Elaboración propia.

\footnotetext{
${ }^{4}$ Las intenciones arquitectónicas que se refieren en este epígrafe provienen de sendas monografías OAB, Ed. Actar, Barcelona, 2015, y Peñín Arquitectos, Biblioteca TC, Ediciones Cuadernos de la Construcción, Valencia, 2014. También han sido reseñadas en distintas revistas especializadas como Arquitectura Viva (135): 40-43, Madrid: Ed. Arquitectura Viva, S. L., 2011.

${ }_{5}^{5}$ Butech, del grupo Porcelanosa, fue contratada por BECSA, adjudicataria del concurso promovido por el ayuntamiento de la localidad.
} 
cerámica vitrificada: no hay buena arquitectura sin un buen cliente, como tampoco la hay sin un buen industrial.

\section{LA PIEZA}

No estamos hablando, por tanto, de un nuevo material o de una investigación puntera en el comportamiento o mejora de los materiales al uso, sino de la manipulación de lo ya existente a través de la implantación de un nuevo sistema. Aquí la materialización de la idea de filtro parte de la reconversión de unas piezas cilíndricas empleadas en los rodillos de determinados hornos de cocción que así, mediante un cambio de dominio, constituyen la base para construir la fachada.

Se emplea un gres porcelánico (-0,1\% de absorción) en cilindros huecos de $5 \mathrm{~cm}$ de diámetro, 93,2 $\mathrm{cm}$ de largo y espesor de pared de $8 \mathrm{~mm}$. La pieza se obtiene mediante extrusión de pasta blanca obtenida a partir de arcillas y otras materias primas inorgánicas amasadas y compactadas. La pasta en estado plástico pasa a través de una matriz formando la sección circular, definida constante en toda su longitud. La extrusora tiene un sistema propulsor, la matriz circular, y la cortadora que recorta las piezas con la constante longitud mencionada (Figuras 4 y 5).

A continuación, la pieza una vez secada, pasa por un proceso de cocción de hasta $1.25^{\circ} \mathrm{C}$ en un horno monoestrato (rodillos en movimiento) y se enfría, transformando sus arcillas en silicatos de aluminio cristalinos sin hidratar. La resistencia mecánica a los agentes químicos, al fuego y la estabilidad dimensional de la pieza se fían a este proceso cuyas variables fundamentales son el ciclo térmico (temperatura-tiempo) y la atmósfera del horno, adaptadas a cada composición y tecnología de fabricación.

Finalmente la pieza se esmalta con una capa de vidriado de entre 75 y 500 micras que la cubre en toda su superficie, otorgándole sus propiedades estéticas: limpieza, brillo, color, textura superficial e implementando las técnicas, como la impermeabilidad y las resistencias químicas y mecánicas.
Para este caso los datos de resistencia mecánica no son determinantes, dado que la pieza es autoportante, siendo el sistema de 8 piezas unidas el que conferirá la resistencia final y las propiedades del conjunto. Las de la pieza quedan reseñadas en la Tabla 1.

Tabla 1. Principales propiedades de la pieza.

\begin{tabular}{|c|c|c|}
\hline \multirow{3}{*}{ Análisis químico } & $\mathrm{Al}_{2} \mathrm{O}_{3} \%$ & $73-75$ \\
\hline & $\mathrm{SiO}_{2} \%$ & $16-18$ \\
\hline & $\mathrm{Fe}_{2} \mathrm{O}_{3} \%$ & $0,3-0,7$ \\
\hline Densidad & $\mathrm{Kg} / \mathrm{dm}^{3}$ & $2,85-2,95$ \\
\hline Porosidad aparente & $\%$ & $14-17$ \\
\hline Agua absorbida & $\%$ & $4,5-5$ \\
\hline Resistencia a flexión en frío & $\mathrm{N} / \mathrm{mm}^{2}$ & $>36$ \\
\hline Dilatación térmica a $1.000^{\circ} \mathrm{C}$ & $\%$ & 0,54-0,59 \\
\hline \multicolumn{2}{|l|}{ Resistencia al choque térmico } & \\
\hline
\end{tabular}

\section{EL SISTEMA}

A partir de la definición de esta pieza la columnilla de 7,5 m de altura total, formada por ocho de ellas, se perfecciona mediante un procedimiento iterativo y empírico. Unos separadores de polietileno resistentes al exterior se colocan entre cada una de ellas a modo de casquillos (Figura 6). De diámetro exterior de $34 \mathrm{~mm}$, interior de $12 \mathrm{~mm}$, una longitud total de $105 \mathrm{~mm}$, dejan una junta entre cilindros cerámicos de $5 \mathrm{~mm}$ mediante un saliente horizontal.

El conjunto se cose a través de una barra de acero liso de 16 $\mathrm{mm}$ de diámetro y 7,6 $\mathrm{m}$ de longitud que queda confinada en el cilindro hueco gracias a la inyección de poliuretano P-404 en el interior de la cerámica, rellenando todo su interior para impedir cualquier tipo de holgura. La barra sirve de guía para la colocación sucesiva por la parte superior de cada una de las piezas cilíndricas y los sucesivos rellenos de poliuretano.

Tras la realización de un prototipo a escala real de la columnilla quedó patente la falta de rigidez y estabilidad del conjunto, produciéndose una oscilación tras solicitación horizontal, de alrededor $30 \mathrm{~cm}$ respecto al eje en su máxima dimensión.
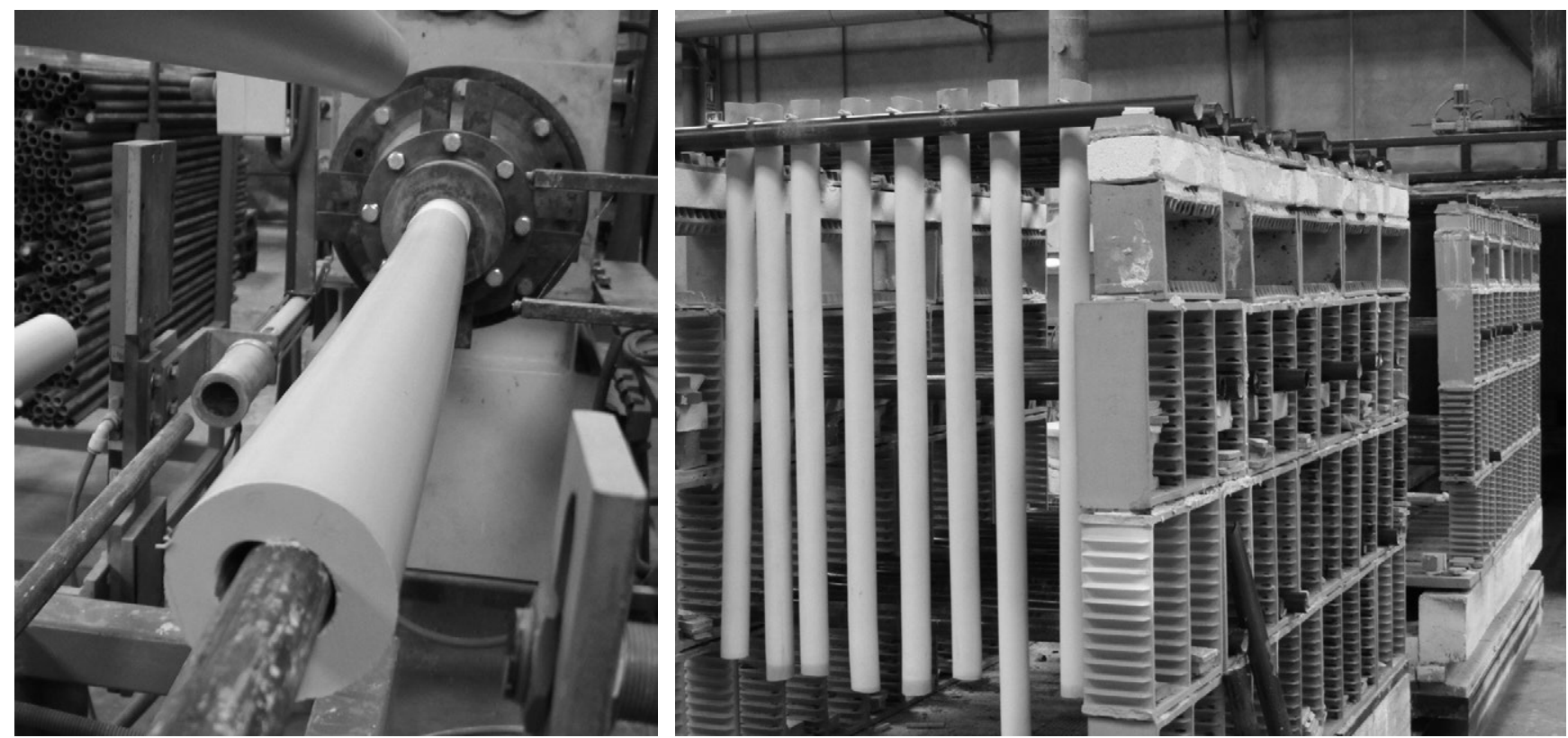

Figuras 4 y 5. Extrusión y secado tras cocción de la pieza en fábrica. Cortesía de Butech. 

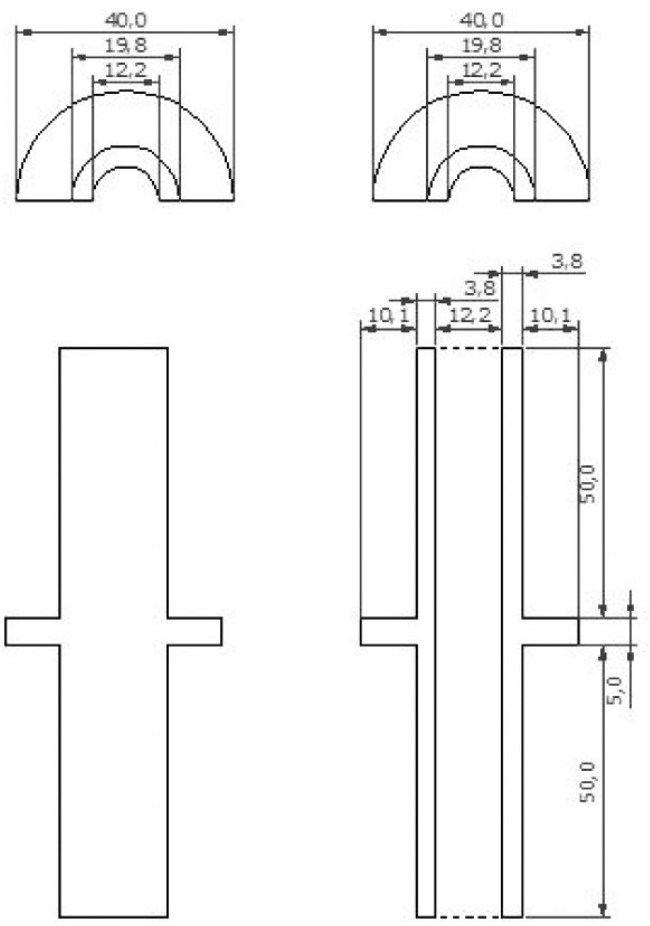

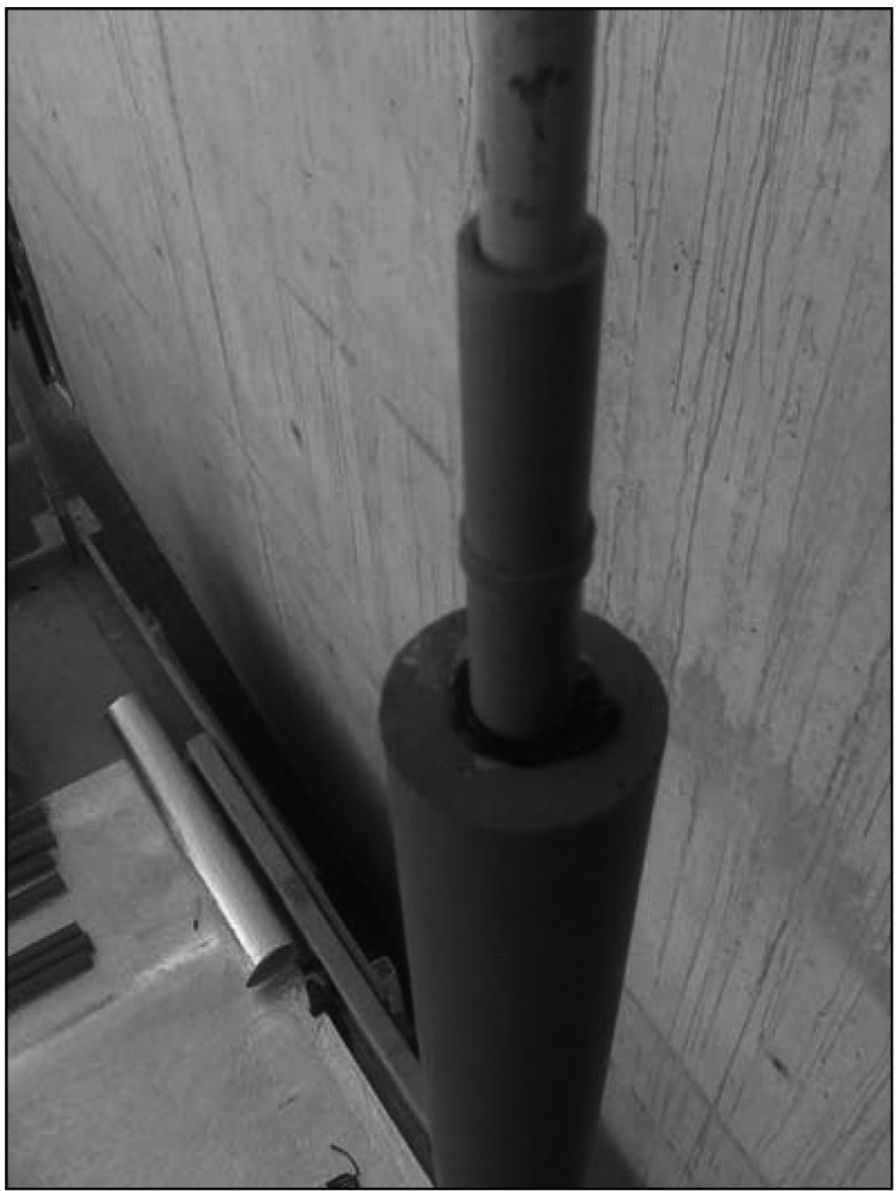

Figura 6. Fase de implementación del sistema en prototipo. Casquillo de polietileno empleado como separador. Cortesía de Butech.

Para corregirla se decidió pretensar la barra de $16 \mathrm{~mm}$ para estabilizar la columnilla e introducir una subestructura trasera unida al edificio que puntualmente arriostrara cada columnilla a la altura de la tercera pieza $(\mathrm{h}=2,80 \mathrm{~m})$. La tensión ejercida con la llave dinamométrica - en torno a $10 \mathrm{~kg}-\mathrm{se}$ introduce fijando por la parte superior mediante tuerca y aplicándola por abajo. La pieza se preinstala en situación de servicio con anclajes en taller para su posterior desmontaje, transporte y colocación definitiva, como muestra la Figura 7.
La introducción de una pletina blanca lacada al fuego, de $3 \mathrm{~mm}$ de espesor y atornillada a un pasamanos lineal gris oscuro - a $15 \mathrm{~cm}$ de la fachada y unido al edificio-, permite reducir la deformación del conjunto y llevarla a dimensiones casi nulas (tolerancia de $2 \mathrm{~mm}$ de desviación respecto al eje) cuando la solicitación se produce a la altura de las personas, como solo así se puede producir. El conjunto de columnillas se fabrica en taller y se transporta en camión a pie de obra.
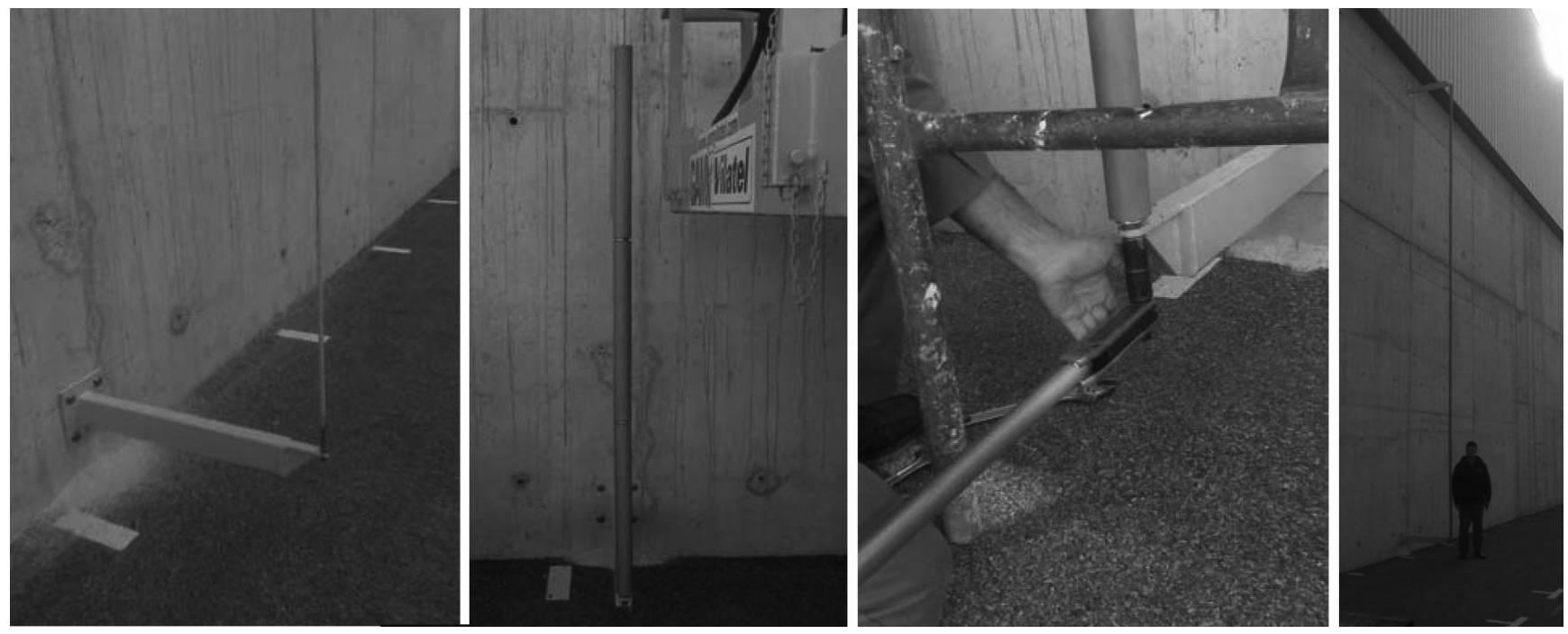

Figura 7. Fases sucesivas de prefabricación de la columnilla en taller. Barra de guía, inserción de pieza, pretensado por la parte inferior mediante llave dinamométrica y situación final. Cortesía de Butech. 

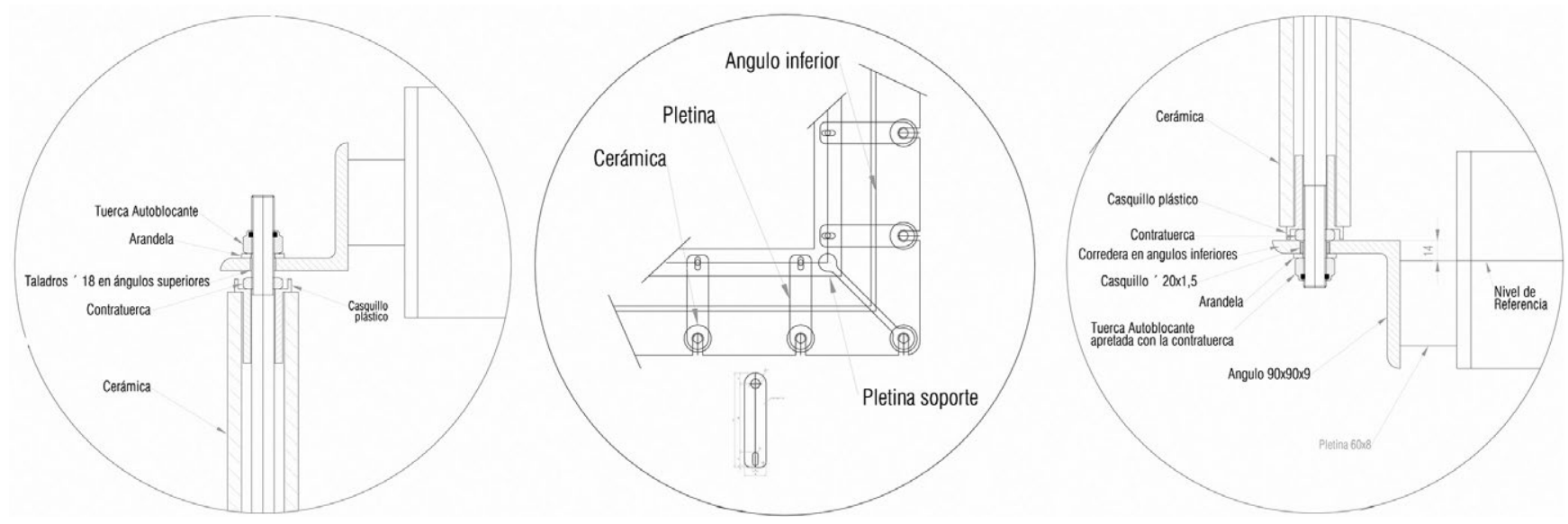

Figura 8. Anclajes inferior, intermedio y superior. Cortesía de Butech.

\section{LA PUESTA EN OBRA}

Una vez resuelta la estabilidad de cada columnilla se idea el sistema de fijación al edificio realizado fundamentalmente a base de elementos de cerrajería.

En primer lugar los dos cantos de las losas en voladizo del edificio, separadas de 7,5 m, se cubren con sendas 'eles' situadas con su pala hacia dentro para la sujeción de las columnillas, y con husillos cortados en taller mediante láser para la inserción de las columnillas. Esta disposición en doble ele invertida no arroja sombra entre columnillas y perfiles, e incrementa la sensación de liviandad del conjunto, aparentemente suspendido por puntos. El citado sistema de eles estándar, conformadas en frío de dimensiones $90 \times 90 \times 9 \mathrm{~mm}$, permite mediante unos separadores formados por pletinas y situados a eje de cada 3 columnillas, soldarse al canto de hormigón de la losa. se fijan sobre una placa de espera que previamente ha dejado la constructora general del edificio para permitir su uso sin interferencias por parte del industrial. Este mecanismo salva las irregularidades del hormigón y limita a parcelas bien diferenciadas las responsabilidades de las empresas que acuden, manipulan e inciden en el detalle (Figura 8).

Las 1.044 columnillas que se instalarán sobre esta perfilería quedan separadas por $19 \mathrm{~cm}$ entre ejes, dejando huecos de 14 $\mathrm{cm}$. La columnilla se insertará por la parte inferior y se fijará mediante una tuerca que queda por la cara oculta de la ele y una contratuerca alojada en el espesor del casquillo de polietileno. Otros dos separadores plásticos completan el detalle.

Una vez fijado por la cara superior, la columnilla se inserta en el husillo abierto de la ele inferior y se fija al superior con un sistema equivalente. A continuación se ajusta la tensión introducida en la barra con llave dinamométrica hasta alcanzar los $10 \mathrm{~kg}$. Finalmente se ejecuta el arriostramiento definitivo que, como ha quedado descrito, se realiza sobre el pasamanos que construye el espacio intersticial, tal y como muestra la Figura 9.

\section{EL EDIFICIO}

La resoluci trasciende la escala de la pieza, de la columnilla y abre caminos para el resto de decisiones proyectuales. Así, detrás de esta membrana cerámica, el edificio replica con paneles 'minionda' que revisten el fondo del espacio intersticial, construyendo su envolvente térmica. Las sombras que arroja la una están alojadas en los otros, pintados de oscuro para enfatizar la profundidad y para despegar todavía más del edificio la fachada (Figuras 10 y 11).

En el interior la presencia de la cerámica se extiende a los intradoses de estos paneles, doblados con ladrillo panal pintado de blanco y de cuidada ejecución. El suelo también es cerámico enmallado en $3 \times 3$, siguiendo una vez más la tradición mediterránea. La carpintería se define y ejecuta en cerrajería de acero pintado en gris, de manera acorde con los sistemas de fijación descritos.

La atmósfera de sencillez, trabajada a través de la integración de las instalaciones, la luminosidad de techos y lucernarios blancos, subraya la presencia exterior del filtro (Figura 12).

Por su parte, la urbanización exterior retoma el tema de la geometría circular tanto en el diseño de las jardineras como en la traza curva del talud, incluyendo luminarias diseñadas para la ocasión de sección cilíndrica recortada. La suave pendiente se tapiza con especies autóctonas como el romero rastrero y, a modo de podio, ensalza y otorga un aire monumental al edificio. La cortina cerámica no sólo queda protegida del

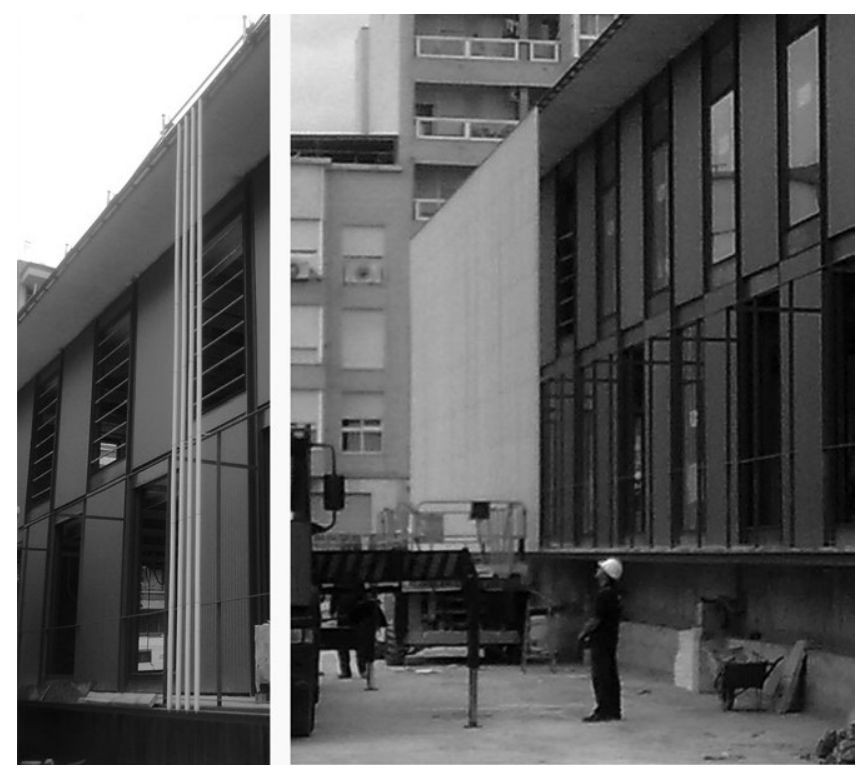

Figura 9. Fotos de puesta en obra tras instalación de ángulos superior e inferior de anclaje y sistema intermedio de arrriostramiento. Cortesía de Butech. 


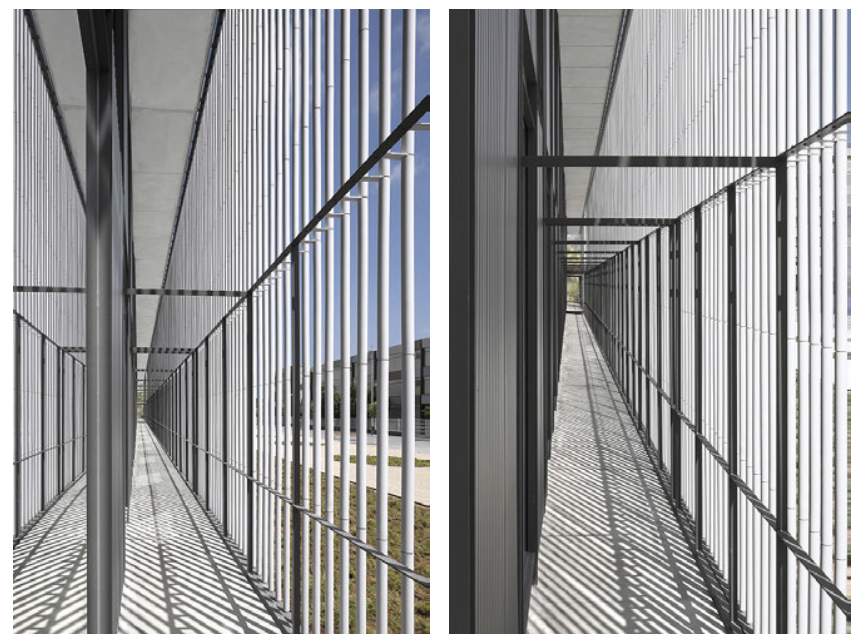

Figuras 10 y 11. El espacio intersticial (3). Elaboración propia y cortesía de Alejo Bagué.

alcance directo desde el exterior, sino que adquiere un cierto carácter de infinito peristilo urbano,lo interrumpido para permitir el acceso y el paso de la plaza a la avenida.

Finalmente, los beneficios de la disposición de esta cortina recuerdan el origen de aquellos filtros de eslabones de los pueblos (Figura 13). Además de suponer un eficaz umbral de intimidad y luz para la lectura logran la creación de un colchón climático, que aquí cuenta con la contribución de la plantación del podio. La humedad provocada por el frecuente regado y la sombra del espacio intersticial logran la disminución de la incidencia térmica en el interior del edificio. La obra y el filtro cerámico fueron recogidos en numerosas publicaciones entre las que destacan además de las monografías referidas Arquitectura Viva (5) o el estudio sistemático de filtros en la plataforma filt3rs.net (6).

\section{EL DISEÑO CON EL INDUSTRIAL}

El procedimiento descrito para la definición de la cortina cerámica se extiende al resto de elementos del edificio, de- sarrollados en su mayor parte en estrecha colaboración con los industriales. La cortina no es un caso aislado en el edificio. Dan muestra de ello las carpinterías de perfilería de acero estándar a las que se insertan gomas para estanqueidad; el diseño estructural específico de la escalera principal donde la barandilla contribuye a la rigidez; las mamparas interiores de separación mediante paneles metálicos tipo 'trámex' colocados inusualmente en vertical, o los propios elementos de mobiliario tanto interior como exterior ${ }^{6}$. Todos ellos recurren a estrategias de diseño y colaboración con la industria que algunos autores como Richard Sennett en su libro El artesano han definido e ilustrado ${ }^{7}$ : proceso frente al mito de la creatividad, cambio de registro, forzado del límite, ambigüedad o creación de dificultad son algunos de los mecanismos que Sennett identifica para una artesanía renovada.

Volviendo a la cita con la que comenzábamos este texto, Renzo Piano definía a través del pensamiento de Prouvé, la noción de microsolar ${ }^{8}$, un procedimiento por el cual la obra se desdobla en múltiples desarrollos con la industria. Lo que los anglosajones llaman fast-tracking, o diseño progresivo, exige de una participación directa del industrial en el proceso de definición al cabo del cual se produce la contratación. Se trata, pues, de una operativa del proyecto, que en el caso de Prouvé informaba sus métodos de trabajo y su actitud empresarial particularmente progresista.

El proceso, desarrollado en forma de taller, se inicia con un dibujo de intención que desencadena la secuencia y lo retroalimenta iterativamente para desembocar en un prototipo. Una vez corregido, mejorado y aprobado se realiza el shop drawing -como pueden ser los detalles de anclaje de la Figura 9- con el cual se contrata y ejecuta. El procedimiento, cuyo escenario fundamental es la obra, se nutre del trabajo de equipo y del estímulo de la iniciativa individual por encima del trabajo mecánico y sectorizado.

Finalmente se produce un doble beneficio, tanto para el propio proyecto como para la solvencia del industrial que ve ampliado su catálogo, recuperando una cierta dimensión colectiva y superando la supuesta unicidad de la obra arqui-

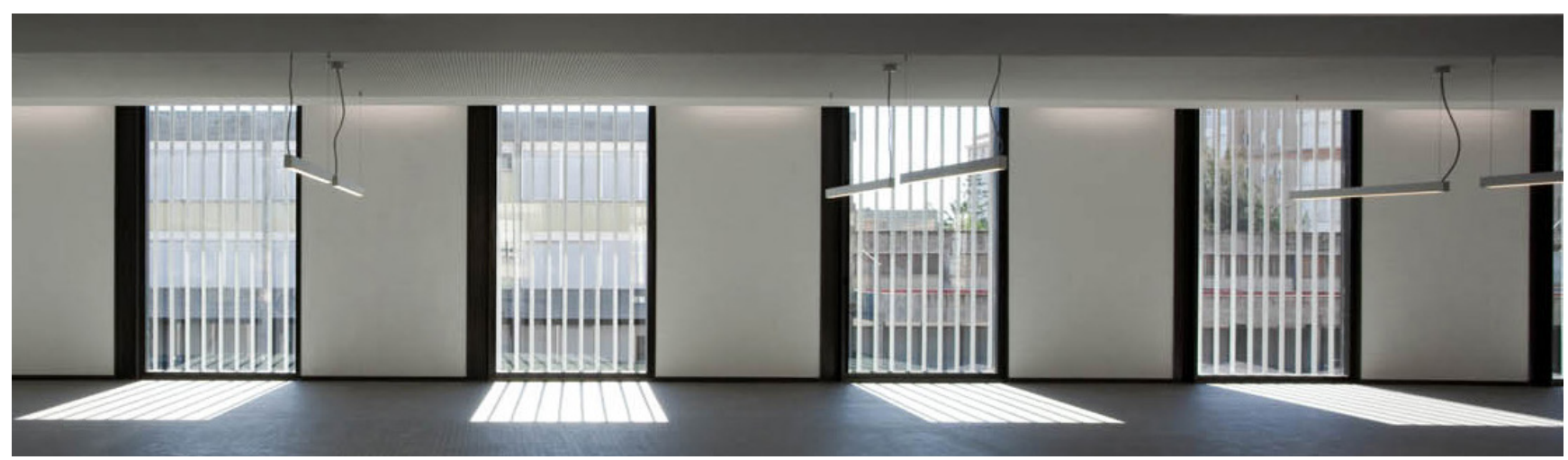

Figura 12. Cortina cerámica desde el interior. Cortesía de Alejo Bagué.

${ }^{6}$ La farola Cortatub fue objeto de un diseño industrial específico patentado junto al industrial MUD.

Por poner un ejemplo, Sennett describe en su libro El artesano (7), cómo Frank Ghery en el Guggenheim de Bilbao, y ante la imposibilidad del uso de la aleación de plomo y cobre que conocía, inició un proceso de innovación con la industria sin final a priori y con cambios de dominio constantes que desembocó en las conocidas planchas de titanio de un tercio de milímetro, paradójicamente mucho más estables que un material masivo como la piedra, y que obligaron a innovar una impermeabilización autocicatrizante para su fijación.

8 Renzo Piano en la entrevista realizada por Antonie Picon en Du plateau Beaubourg au centre Georges Pompidou, Editions du Centre Pompidou, Paris, 1987 (9). 


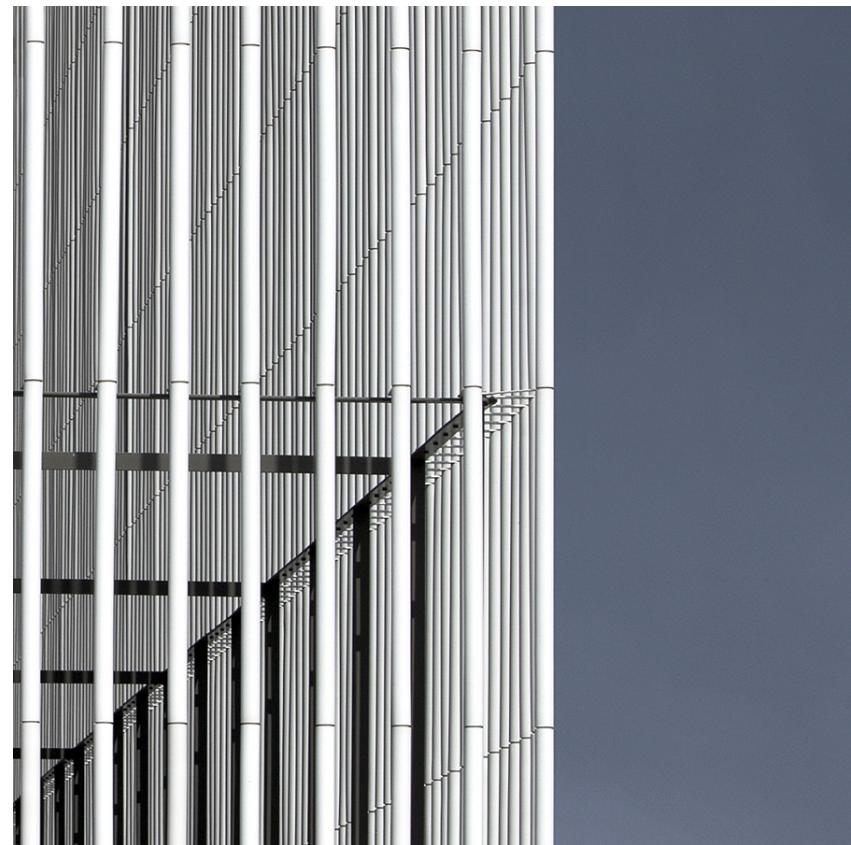

Figura 13. Detalle exterior (4). Cortesía de Diego Opazo.

tectónica. Los ejemplos en el Pompidou son numerosísimos, bastaría señalar el desarrollo de Piano junto a OTIS de un ascensor que pasó de ser imposible a ser una de las referencias del catálogo de la empresa. El arquitecto se desmarca deliberadamente de la mitificación arrogante del acto creativo del movimiento moderno para identificarse con los sistemas de producción y fabricación como indicábamos en la tesis $L a$ transformación del proyecto arquitectónico durante el proceso constructivo (8).

En el caso que nos ocupa, tras la finalización de las obras se produjo la incorporación de la Lama tubular en el catálogo de Butech-Porcelanosa ${ }^{9}$, como también ha sucedido en otros ejemplos de la trayectoria de OAB. No se trata, pues, de un hecho aislado, lo cual revela la condición estratégica de la participación del industrial en su arquitectura, y nos ayuda a inscribir el proyecto de la Biblioteca en una determinada cultura arquitectónica ${ }^{10}$. Mencionaremos tan sólo, por su parecido con la Biblioteca tanto en la forma como en el proceso, el caso de la habilitación de la Roca Gallery de Barcelona, donde se desarrolló coordinadamente junto a dos industriales, vidriero y cerrajero, un innovador sistema de paneles formados por vidrios colocados transversalmente a la fachada ${ }^{11}$ (Figura 14).

Esta pequeña investigación propone nuevamente un cambio de dominio; un material conocido puesto en obra de manera distinta, sin un resultado predeterminado y con el obligado concurso del industrial, como ya hemos descrito en la Biblioteca de Vila-real.

\section{EPÍlOGO. CONTRATACIÓN Y DIRECCIÓN DE OBRA}

La defensa de este planteamiento implica una reflexión sobre los marcos administrativo y legal de las obras en nuestra actual regulación. Obligaría a establecer unos plazos de proyecto más extensos en el tiempo, permitiendo el desarrollo con el industrial, o bien a considerar la obra como un territorio donde el proyecto sigue vivo, algo que hoy bien podría ser considerado escandaloso. En el caso del Pompidou -que se muestra como un ejemplo paradigmático de esta manera de hacer, también en lo legal- la aplicación de la ley del ingeniero ${ }^{12}$ no sólo atribuía responsabilidades en función de los resultados de la obra, sino también establecía un procedimiento de contratación específico y novedoso: el managment contractor. Consistía en la contratación por parte de la propiedad de una única empresa constructora ${ }^{13}$, a un precio fijo y cerrado que no interviene en el trabajo constructivo para no generar conflictos de intereses, y subcontrata y coordina todos los industriales. Su trabajo práctico se basaba en preparar licitaciones y contratos a partir de la documentación suministrada por los técnicos, y en realizar un seguimiento y vigilancia de la obra incluido su control de calidad, desvinculado de los profesionales. En el caso de Vila-real la contratación directa de Butech por parte de la propiedad con la única supervisión administrativa de la constructora facilitó el
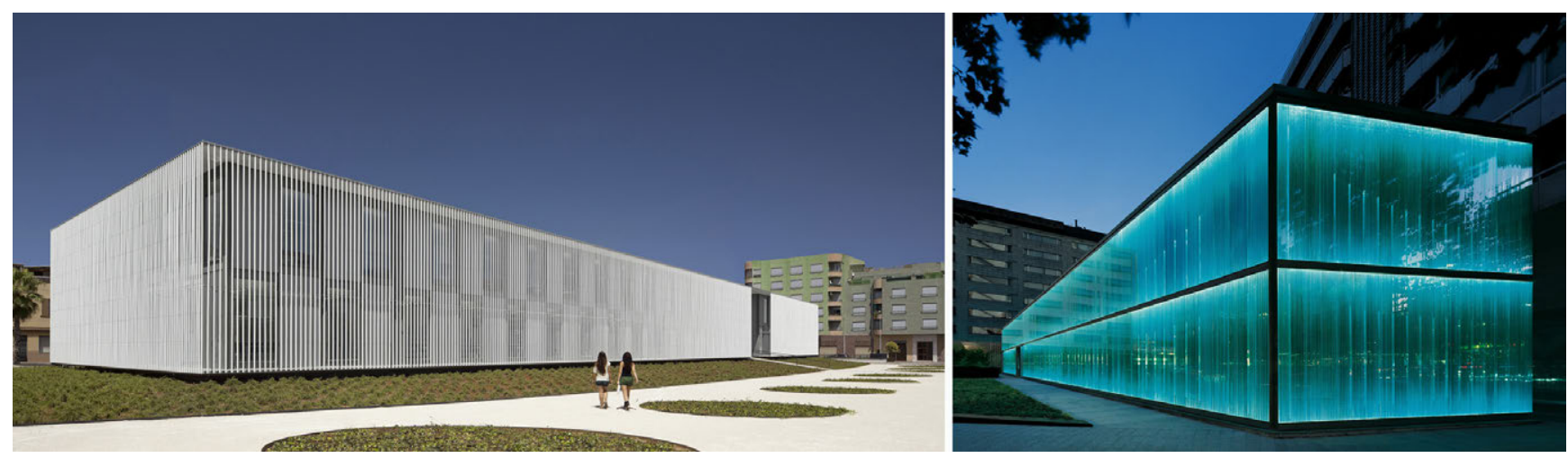

Figura 14. Biblioteca de Vila-real vs. Roca Gallery de Barcelona. Cortesía de Alejo Bagué.

\footnotetext{
${ }_{9}$ Butech-Porcelanosa incluyó en su catálogo 2012 bajo el nombre Sistema cortina cerámica a base de lamas tubulares.

10 Aquella en la que la teoría emerge de la praxis y que se expresa también en el ámbito académico no sólo en la llamada Escuela de Barcelona, sino también en experiencias ya consolidadas como la Cátedra Blanca de Barcelona o la editorial Palimpsesto.

${ }^{11}$ Ferrater, Borja, Carlos y Lucía. Roca Gallery, 2008-2009.

${ }^{12}$ Un sistema de honorarios que penalizaba los desajustes respecto al presupuesto a partir de una desviación del $12 \%: 8,78$ \% si desvío < $12 \%$; $7,31 \%$ si $12<d<25 \% ; 4,59 \%$ si $d>50 \%$. Porcentajes sobre la previsión.

${ }^{13}$ En el caso del Pompidou fue Grands Travaux de Marseille (G. T. M.).
} 
buen desarrollo de la cortina cerámica. El papel del arquitecto trasciende la prescripción e incluye una importante labor estratégica y de síntesis, incorporando la coordinación de los distintos industriales en el diseño.

Es evidente que el sistema de contratación tiene una especial relevancia a la hora de permitir, ordenar y sistematizar estas definiciones progresivas de la arquitectura, manteniendo el necesario control económico. Es necesaria una visión conjunta y sintética del proyecto, contraria a su división en partidas estancas y a la lógica en ocasiones perversa de las contrataciones privadas y públicas tan frecuentes en nuestro país. La irrenunciable lógica del plazo y beneficio no debe ser contradictoria con la calidad, unicidad y cohe- rencia del proyecto. Sus límites se diluyen y se extienden más allá de su consideración administrativa que culmina en el proyecto de ejecución, cabalgando en un sentido o en otro, sobre la dirección de obra. Seamos claros: no se puede deslindar el proyecto de la dirección de obra si se aspira a la calidad.

La ley de contratos del Estado y el conjunto del marco legislativo del sector de la construcción ${ }^{14}$ debe ponerse al día no sólo para propiciar, sino también para estimular, como toda legislación con altura de miras debe procurar, casos como el de la Biblioteca de Vila-real en los que, en aras de la calidad y del acervo colectivo, el diseño sea el fruto de la integración de industriales y proyectistas.

\section{REFERENCIAS}

(1) Piano, R. (1977). La Technologie n'est pas toujours industrielle, Architecture d'aujourd'hui (189): 51-54. París.

(2) (2001). Jean Prouvé constructeur. París: Editions de la Réunion des Musées Nationaux. Catálogo.

(3) Ferrater, C. (2015). Biblioteca de Vila-real. En $O A B$. Barcelona: Ed. Actar.

(4) Peñín, A. Biblioteca de Vila-real. En Peñín Arquitectos. Valencia: General Ediciones de Arquitectura.

(5) (2011) Cortinaje cerámico, Arquitectura Viva (135): 40-43. Madrid: Ed. Arquitectura Viva, S. L.

(6) Filters. (2012). http://filt3rs.net/photo/reja-ceramica.

(7) Sennet, R. (2009). El artesano. Barcelona: Ed. Anagrama.

(8) Peñín, A. (2007). La transformación del proyecto arquitectónico durante el proceso constructivo (tesis doctoral no publicada). Barcelona: UPC.

(9) Picon, A. (1987). Du plateau Beaubourg au centre Georges Pompidou. París: Editions du Centre Pompidou.

\footnotetext{
${ }^{14}$ Es obligado citar aquí la reciente aprobación del Proyecto de Ley de Arquitectura en Cataluña el 4 de agosto de 2015.
} 